Check for updates

The BMJ

Cite this as: $B M J 2021 ; 375: \mathrm{n} 2481$ http://dx.doi.org/10.1136/bmj.n2481 Published: 11 October 2021

\section{Covid-19: Antibody levels fall after second Pfizer dose, but protection against severe disease remains, studies indicate}

\author{
Elisabeth Mahase
}

Six months after the second dose of the Pfizer-BioNTech covid-19 vaccine, antibody concentrations in healthcare workers had decreased substantially, especially among older men and immunosuppressed people, a study has found. ${ }^{1}$

The Israeli researchers carried out a six month longitudinal prospective study that involved monthly antibody testing of around 4000 vaccinated healthcare workers. At six months the mixed-model analysis showed decreases in antibody concentrations of $38 \%$ for IgG antibodies and $42 \%$ for neutralising antibodies among those aged 65 years or older, when compared with participants aged 18-45. Decreases of $37 \%$ (IgG) and 46\% (neutralising) were also seen in men 65 years of age or older when compared with women in the same age group.

The study, published in the New England Journal of Medicine, reported that immunosuppressed people had decreases in antibody concentrations of $65 \%$ (IgG) and 70\% (neutralising) compared with participants without immunosuppression, while people with a BMI of 30 or over had $31 \%$ higher neutralising antibody concentrations, when compared with those whose BMI was under 30.

The researchers said that IgG antibody concentrations decreased at a consistent rate, while the neutralising antibody concentrations fell rapidly for the first three months after the second dose, followed by a slower decrease between three and six months.

They noted that, in comparison with other vaccines, such as those against measles, mumps, and rubella, there was a much more significant and rapid decrease in antibody concentrations after the Pfizer covid-19 vaccine.

The study's limitations included that it involved only healthcare workers, who were mostly healthy, and so it may not be representative of the wider population. Additionally, it did not look at covid-19 cases, only antibody levels.

\section{Lasting protection against severe illness}

A second study, also published in New England Journal of Medicine, looked at the effectiveness of the Pfizer vaccine against SARS-CoV-2 infection and disease in Qatar. ${ }^{2}$

These researchers found that effectiveness peaked at $78 \%$ in the first month after the second dose before declining gradually, with the decline accelerating after the fourth month to reach $20 \%$ in months five to seven.

However, looking at effectiveness against any severe, critical, or fatal cases of covid-19, the researchers reported that it peaked at around $96 \%$ in the first two months after the second dose and then remained around this level for six months.

To carry out the study, the researchers matched people with positive PCR results to PCR negative persons, according to sex, a 10 year age group, nationality, reason for PCR testing, and calendar week of PCR testing. Using the national covid-19 database, they looked at cases between 1 January and 5 September 2021. A total of 8203 breakthrough infections had been recorded among participants who received one dose of the Pfizer vaccine, and 10 543 infections had been recorded among participants who received two doses.

The researchers concluded that although overall protection against infection waned rapidly one month after the second dose, protection against admission to hospital and death "persisted at a robust level" for at least six months after the second dose.

The paper acknowledged several limitations, including that individual data on pre-existing conditions were not available, only a small proportion of the study population may have had serious coexisting conditions, and only $9 \%$ of the population was aged over 50. As such, the findings would not be generalisable to other countries where elderly people constitute a bigger proportion of the total population.

Levin EG, Lustig Y, Cohen C, etal. Waning immune humoral response to BNT162b2 covid-19 vaccine over 6 months. N Engl J Med 2021 doi: 10.1056/NEJMoa2114583. pmid: 34614326

2 Chemaitelly $\mathrm{H}$, Tang P, Hasan MR, etal. Waning of BNT162b2 vaccine protection against SARS-CoV-2 infection in Qatar. N Engl J Med 2021. doi: 10.1056/NEJMoa2114114. pmid: 34614327

This article is made freely available for use in accordance with BMJ's website terms and conditions for the duration of the covid-19 pandemic or until otherwise determined by BMJ. You may use, download and print the article for any lawful, non-commercial purpose (including text and data mining) provided that all copyright notices and trade marks are retained. 